\title{
Message from the P\&FM Editors to our ad hoc Reviewers
}

This is an Open Access article distributed under the terms of the Creative Commons Attribution Non-Commercial License (https:// creativecommons.org/licenses/ by-nc/4.0/).

\section{ACKNOWLEDGMENT TO REVIEWERS}

All papers submitted for publication in Precision and Future Medicine (P\&FM) undergo peer review. $P \& F M$ papers have at least two ad hoc reviewers. We highly appreciate $P \& F M$ ad hoc reviewers listed below. Listed below are the reviewers who completed reviews up to December 2019. All reviewers gave feedback and guidance on previous P\&FM manuscripts. They largely represent the voice and opinion of $P \& F M$ about articles and gave direction to P\&FM authors for further improvement. Without their support, we would be unable to maintain the quality of the papers in P\&FM, because P\&FM covers various fields, including translational drug discovery, precision medicine, regenerative medicine, and novel diagnostic methods and medical devices.

$\begin{array}{lll}\text { Cheol-In, Kang } & \text { Jung-Ryul, Kim } & \text { Sung Noh, Hong } \\ \text { Dongryul, Oh } & \text { Jungwook, Hwang } & \text { Sung Yong, Oh } \\ \text { Eun Hee, Kim } & \text { Kwang-Yeol, Park } & \text { Tae Jun, Kim } \\ \text { Geun Dong, Lee } & \text { Kyu Yeon, Hur } & \text { Tae Sung, Kim } \\ \text { Gyeong-Moon, Kim } & \text { Mi Ae, Jang } & \text { Tae Wook, Kang } \\ \text { Hee Jung, Son } & \text { Mi Ji, Lee } & \text { Tong In, Oh } \\ \text { Ho jae, Bae } & \text { Mira, Park } & \text { Won-Jung, Koh } \\ \text { Ho Jun, Seol } & \text { Mirae, Lee } & \text { Woo-Keun, Seo } \\ \text { Ho Yeong, Lim } & \text { Myoung-Hwan, Ko } & \text { Yeo Jin, Kim } \\ \text { Hong Ryull, Pyo } & \text { Myung-Jin, Chung } & \text { Yesel, Kim } \\ \text { Hyuk, Lee } & \text { Myung-Ju, Ahn } & \text { Yon Ju, Ryu } \\ \text { Hyung Jun, Kim } & \text { Sang Yun, Ha } & \text { Yong Han, Paik } \\ \text { Jaehwan, Kim } & \text { Se hoon, Lee } & \text { Yong Il, Shin } \\ \text { Jeon Yeob, Jang } & \text { Se Hoon, Park } & \text { Yong Jae, Kim } \\ \text { Jihyung, Park } & \text { Semin, Chong } & \text { Yong Kee, Kim } \\ \text { Jin Oh, Choi } & \text { Semin, Lee } & \text { Yong-Soo, Kwon } \\ \text { Jin San, Lee } & \text { Seong-Beom, Koh } & \text { Yongwan, Lim } \\ \text { Jin Young, Youn } & \text { Seung Hoi, Koo } & \text { Yoon Kyung, Kim } \\ \text { Jiwon, Hwang } & \text { Seung Tae, Kim } & \text { Young kyu, Moon } \\ \text { Jong Mu, Sun } & \text { Silvia, Park } & \text { Young min, Eun } \\ \text { Jong Won, Chung } & \text { Soo-Jin, Kim } & \text { Young Saing, Kim } \\ \text { Jong Won, Kim } & \text { Soon Il, Lee } & \text { Youngyih, Han } \\ \text { Jong Wook, Chang } & \text { Su Jin, Lee } & \text { Yu-Shik, Hwang } \\ \text { Ju-Hong, Min } & \text { Suk-Kyung, Lim } & \\ \text { Jun Ho, Jang } & \text { Sung A, Chang } & \end{array}$

Oh Young Bang

Editor-in-Chief

Precision and Future Medicine 